\title{
ARTICLE \\ Research on Auto-Exposure Algorithm Based on Image Big Data and Information Entropy
}

\section{Yunfei Bai*}

Tianjin Big Data Management Center, Tianjin, 300000, China

\begin{tabular}{|c|c|}
\hline ARTICLE INFO & ABSTRACT \\
\hline Article history & An Auto-Exposure (AE) algorithm based on image big data and infor- \\
\hline Received: 8 August 2019 & mation entropy is proposed. On the basis of the traditional algorithm for \\
\hline Revised: 9 August 2019 & automatic exposure adjustment based on image brightness, image big data \\
\hline Accepted: 10 October 2019 & bient luminance evaluation and image information entropy, the dimension \\
\hline Published Online: 16 October 2019 & $\begin{array}{l}\text { of information acquisition of the automatic exposure system is improved, } \\
\text { thus improving the image effect and scene adaptability of the camera. } \\
\text { Especially in high dynamic range scenes, compared with the traditional }\end{array}$ \\
\hline Keywords: & algorithm, the effect is significantly improved. \\
\hline
\end{tabular}

AE

Big data

Information entropy

Ambient luminance

\section{Introduction}

$\mathrm{W}$ ith the rapid development of today's society and the progress of science and technology, the need to ensure social security and the safety of people's travel has become increasingly prominent, and the intelligent security industry has gradually entered into people's vision. As the main front-end acquisition equipment in the field of security monitoring, digital cameras are the foundation and premise of all subsequent security functions. Only by continuously improving the image effect and collecting more abundant information can the functions of back-end image analysis and identification be effectively realized. As the core function of determining the image effect, the $\mathrm{AE}$ of the camera occupies an increasingly important position. The principle of AE is to dynamically control and adjust the camera's exposure time, gain of the camera according to the brightness information of the digital image received by the image sensor, so as to achieve the image brightness effect suitable for human eyes' senses. The feasibility of the AE algorithm described in this paper has been fully verified in cameras in the field of intelligent traffic and monitoring such as the solution of the Hisilicon 3559A processor matched with Sony CMOS sensor IMX305, and remarkable results have been achieved.

\footnotetext{
*Corresponding Author:

Yunfei Bai (1984-),

Male, a native of Wuyi County, Hebei Province, China, graduate degree;

Research direction: digital image processing, deep learning, big data;

Correspondence address: Tianjin Big Data Management Center, No. 2 Guangshun Road, Hexi District, Tianjin, 300000, China; E-mail: jamesxd5033@hotmail.com.
} 


\section{Research Background}

For the AE algorithm of the camera, the traditional research direction is based on the average brightness of the image $^{[1]}$ or the weighted average brightness based on the region of interest $(\mathrm{ROI})^{[2]}$, as the photometric reference of the image, or based on the method of dynamic adjustment of the image information entropy to obtain the maximum value $^{[3]}$ as the target of AE. However, camera equipment in the field of intelligent security is widely used in indoor and outdoor scenes with various brightness, contrast and dynamic range, and the above methods have obvious problems in scene adaptability.

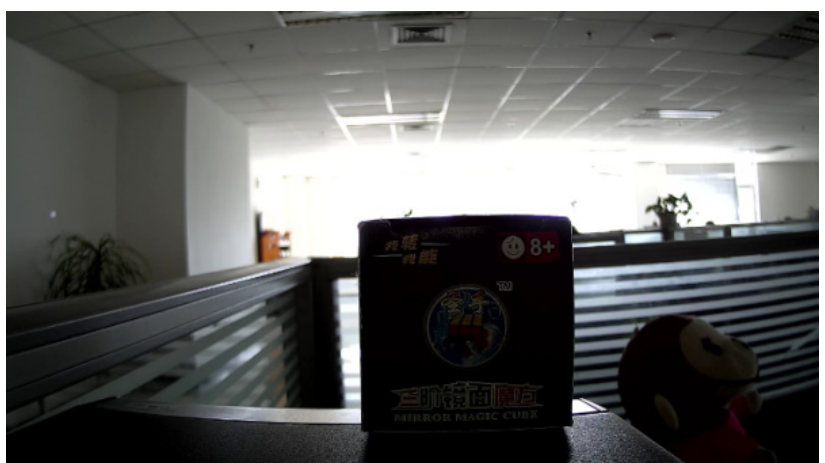

(a) $\mathrm{AE}$ effect of average brightness statistics

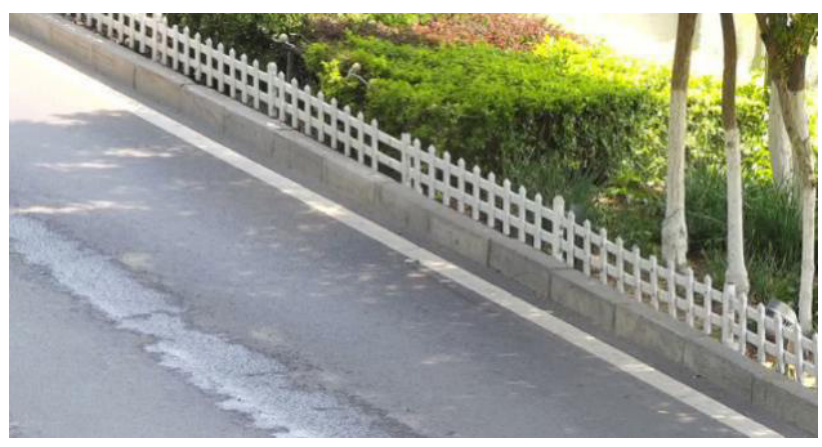

(b) AE effect of ROI weighted brightness statistics

Figure 1. Defects of Traditional AE Mode Based on Image Brightness in Different Scenes

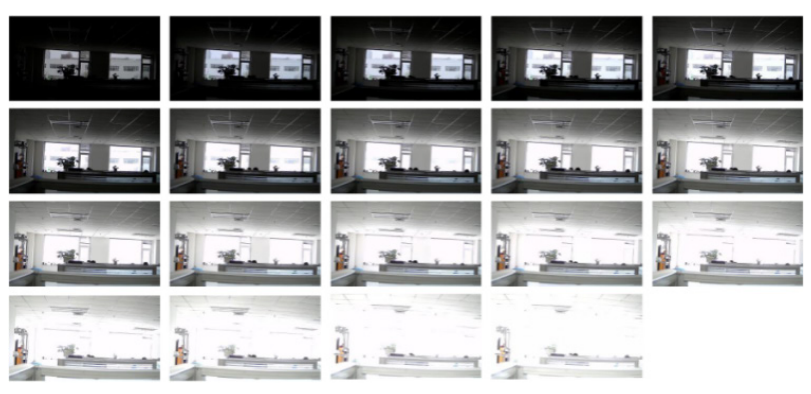

(a) Pictures of different exposures in the same indoor scene

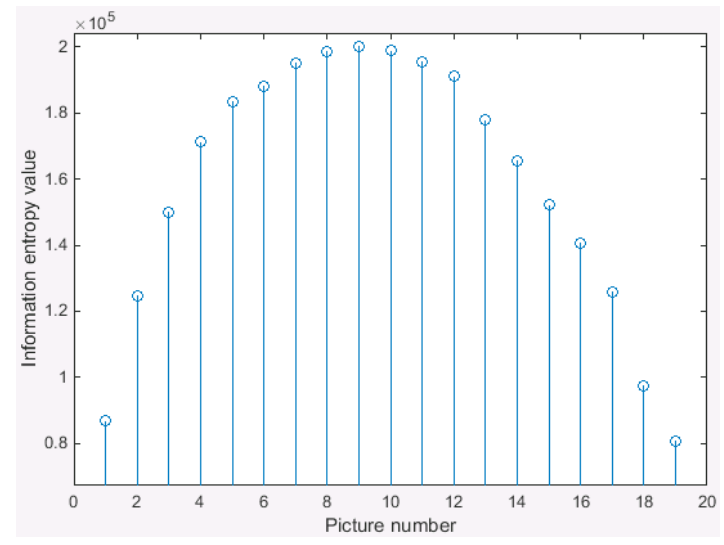

(b) Information entropy corresponding to the picture

Figure 2. Statistical Results of Traditional AE Algorithm Based on Image Information Entropy

Figures 1 and 2 are typical scene pictures captured by current security cameras. Figure 1(a) shows the effect of $\mathrm{AE}$ algorithm using the global average brightness mode. In a high dynamic range scene, the average brightness of the image is increased due to the high brightness of the background, the foreground picture is too dark, and the details are difficult to be clearly displayed. Figure 1(b) shows the effect of AE algorithm based on ROI weighted brightness statistics. Since the pavement texture is rich, it is selected as the area with larger ROI weight, which is the main reference for AE adjustment. Moreover, due to the fact that the road surface is in the shadow range, the average brightness of ROI is low, therefore, the overall brightness of the image is improved, resulting in serious overexposure of background plants and loss of effective information. Figure 2(a) shows the images captured in an indoor monitoring scene with the exposure value from low to high. Figure 2(b) shows their corresponding image information entropy. According to the traditional information entropy maximum algorithm, the ninth picture in figure 2 (a) should theoretically be the optimal effect of AE adjustment, but in the actual use process, after the longterm testing, the seventh picture takes into account both the indoor scene and the information outside the window, and is most in line with human eyes' senses in terms of brightness and visual effect. In other words, in terms of visual effect, the image of the maximum information entropy is not necessarily the most suitable for human eyes' senses.

In addition, image big data has been gradually applied in the image processing of the traffic field, which is mainly reflected in the extraction of illegal or violation information from captured images, thus maintaining urban 
traffic safety ${ }^{[4]}$. However, there are still some gaps in the fields of mining images themselves and the combination of $\mathrm{AE}$ algorithm and big data.

\section{Methods}

Aiming at the adaptability defects of the traditional AE algorithm, this paper proposes an $\mathrm{AE}$ algorithm based on image big data and information entropy. The basic idea of this algorithm is to raise the dimension and add the ambient luminance evaluation index on the basis of the evaluation of the image by the traditional $\mathrm{AE}$ algorithm, so as to dynamically adjust the brightness weight of each gray level pixel in the image histogram area and obtain the weighted image brightness. At the same time, using the information entropy evaluation method ${ }^{[5]}$ and the analysis and mining of large image data, the optimal parameters are obtained. The brightness of the set $\mathrm{AE}$ target is adjusted, and then the magnitude and direction of exposure are determined according to the relationship between image brightness and target brightness, thus achieving the purpose of AE adjustment. We have collected about 3 million pictures captured by monitoring and intelligent traffic cameras in different locations across the country under different climates, environments and light intensities, forming a large data set of pictures for typical scenes of intelligent security monitoring for the determination of subsequent algorithm parameters. The basic flow of the algorithm is shown in Figure 2.

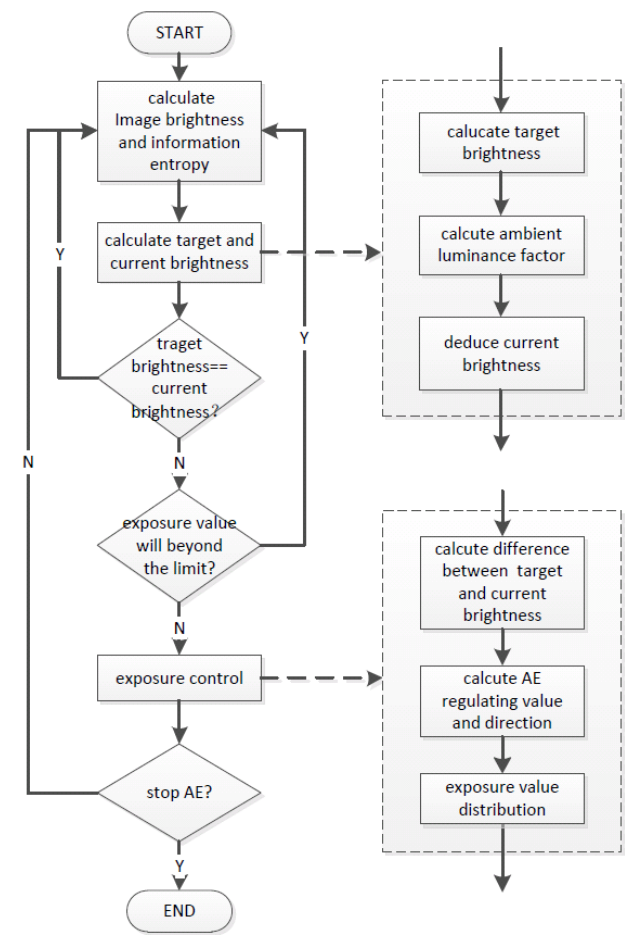

Figure 2. Algorithm flow chart
First, based on the continuity of camera frame information and illumination ${ }^{[6,7]}$, the information of the Nth frame can be used as the basis for calculating the exposure value of the $\mathrm{N}+1$ th frame. From the image brightness histogram hist, the probability $\mathrm{p}_{(i)}$ of obtaining the i-th histogram is:

$$
\mathrm{p}_{(i)}=\operatorname{hist}_{(i)} /(\mathrm{h} * \mathrm{w})
$$

In an 8-bit digital image system, the value range of $i$ is from 0 to $255, \mathrm{~h}$ and $\mathrm{w}$ are the height and width of the image respectively, and then the information entropy E of the frame image is obtained according to the information entropy calculation formula:

$$
\mathrm{E}=\sum_{i=0}^{255}\left(-\mathrm{p}_{(i)}\right) * \log \left(\mathrm{p}_{(i)}\right)
$$

The average brightness of an image can be obtained by the following formula:

$$
\mathrm{L}_{\mathrm{avg}}=\sum_{i=0}^{255}\left(i * \operatorname{hist}_{(i)}\right) /(\mathrm{h} * \mathrm{w})
$$

Image information entropy can reflect the richness of a frame of image information. In theory, the human visual effect should be optimal for the frame with the largest information entropy. However, through the analysis of large image data and the contrast test between image information entropy and human eyes' senses, we have come to the conclusion that there is a certain deviation between the information entropy optimal image and the human eyes' senses optimal image. This is because the image information entropy is greatly affected by the image texture richness, noise and other factors ${ }^{[8]}$, while the human eye is less affected.

Based on the mining and sorting of large image data, we have made a series of attempts in the direction of $\mathrm{AE}$ adjustment guided by the mixture of target brightness and information entropy. Finally, we have worked out an optimization scheme, which is based on the image information entropy $\mathrm{E}$, the new image target brightness is obtained according to the system target brightness input from outside and the image information entropy threshold value :

$$
\mathrm{L}_{\text {tar }}=\mathrm{L}_{\text {in }}-l_{\text {unit }} * \max \left(0,\left(\frac{\mathrm{E}-\mathrm{E}_{\text {th }}}{\mathrm{E}_{\text {uint }}}\right)\right)
$$

In the formula, is the weight of target brightness compensation, is the unit of image information entropy calculation and is the target entropy value. In order to reduce the number of parameters, is uniformly set to 128 at the time of data capture to keep the image from being darker in each scene. Based on formula (4), it could obtain that 


$$
\mathrm{L}_{\mathrm{tar}}=\mathrm{L}_{\mathrm{in}}-\max \left(0,\left(\frac{\mathrm{E}-\mathrm{E}_{\mathrm{th}}}{\mathrm{t}}\right)\right)
$$

Among them, $\mathrm{t}=\mathrm{E}_{\text {uint }} / l_{\text {unit }}$. The determination of the above parameters is completed through the strategy of data iteration. Based on the big data set of images of typical scenes in intelligent security, we first carry out pre-processing operations such as image scaling to reduce the image size and facilitate processing while preserving histogram information. Then, according to the optimal effect periods of different cameras, the data set is divided into two subsets A and B, A is the collection of captured pictures within the optimal effect period of each camera, with about 1.8 million pictures and $\mathrm{B}$ is the set of pictures to be optimized, with about 1.2 million pictures. In the data collection, 20,000 pictures are randomly selected to form sub-data sets $a$ and $b$ as initial data sets for image analysis. After the data set is determined, the images in the set are first analyzed to obtain the target information entropy $E_{a}$, and then the data set $b$ is mined and sorted to start the iteration of parameters. The specific process is as follows: For recording each picture $\mathrm{L}_{\text {in }}$ in the $\mathrm{b}$ set, the initial value of $t$ is set to $1 / 10000$, and the initial value $E_{\text {th }}$ is set to $E_{a}$, and substitute each parameter into formula (5) to obtain a new $\mathrm{L}_{\text {tar }}$. As the exposure time and gain of the image are approximately linear with the brightness of the image, we use python language to perform linear processing in pixel domain for the picture in $\mathrm{b}, \mathrm{L}_{\mathrm{tar}}$ is the brightness of the image. As the next iteration, $\mathrm{L}_{\text {tar }}$ is as $\mathrm{L}_{\mathrm{in}}$. The $\mathrm{L}_{\text {tar }}$ difference between the previous and the following two iterations is gradually reduced through the iterative process of adjusting the parameters, so as to achieve the purpose of convergence. In the process of convergence, the parameters are substituted into data set a for correction of $E_{\text {th }}$ after each of the iteration. After the evaluation function has fully converged, gradually increase the number of samples of data sets $a$ and $b$ and fine-tune the parameters until the function convergence requirements of the whole data sets A and B are met. According to our experimental results, the parameters begin to converge in the second round of iteration and reach basic stability in the seventh round. Finally, $l_{\text {unit }}=2, \mathrm{E}_{\text {uint }}$, is 3500 , and $\mathrm{E}_{\text {th }}$ is $2.9 * 10^{5}$, which are taken as the fixed parameter of formula (4) for the calculation of target brightness.

Next, according to the exposure time $\mathrm{S}$ and gain $\mathrm{G}$ of the current system, using the input target brightness $\mathrm{L}_{\text {in }}$ and fixed basic target brightness $\mathrm{L}_{\text {base }}$ of the system, as well as the maximum exposure time $\mathrm{S}_{\max }$ and maximum gain value $\mathrm{G}_{\max }$ of the system, the external environment brightness proportion value of the current frame can be obtained:

$$
\mathrm{f}_{\mathrm{t}}=\mathrm{S} * \mathrm{G} *\left(\mathrm{~L}_{\text {in }} / \mathrm{L}_{\text {base }}\right) /\left(\mathrm{S}_{\max } * \mathrm{G}_{\max }\right)
$$

The $\mathrm{L}_{\text {base }}$ here is 100 . Since the exposure time $\mathrm{S}$ cannot be 0 , it can be seen from the formula that the value range of $f_{t}$ is $(0,1]$. The larger the $f_{t}$ is, the smaller the brightness of the current scene is. According to the brightness coefficient $f_{t}$ of the external environment, the ambient luminance factor $f_{\text {env }}$ in the current scene is obtained:

$$
\mathrm{f}_{\text {env }}=\left\{\begin{array}{c}
\mathrm{f}_{\min }, \quad \mathrm{f}_{\text {env }} \leq \mathrm{f}_{\min } \\
\mathrm{f}_{\mathrm{t}}, \quad \mathrm{f}_{\min }<\mathrm{f}_{\text {env }}<\mathrm{f}_{\max } \\
\mathrm{f}_{\max }, \mathrm{f}_{\text {env }} \geq \mathrm{f}_{\max }
\end{array}\right.
$$

$f_{\min }$ and $f_{\max }$ indicate the upper and lower limits of the $\mathrm{f}_{\text {env }}$ values, taking 0.3 and 0.7 respectively in practical application. For brightness histogram, the lower the ambient luminance is, the greater the weight of darker part of the image should be to promote the AE system to adjust in the direction of increasing the image brightness. On the contrary, the greater the ambient luminance is, the greater the weight of highlighted part of the histogram should be. As shown in figures 4 (a) and 4 (b), for the scenes with dark and bright ambient luminance, there are histogram brightness weight functions and :

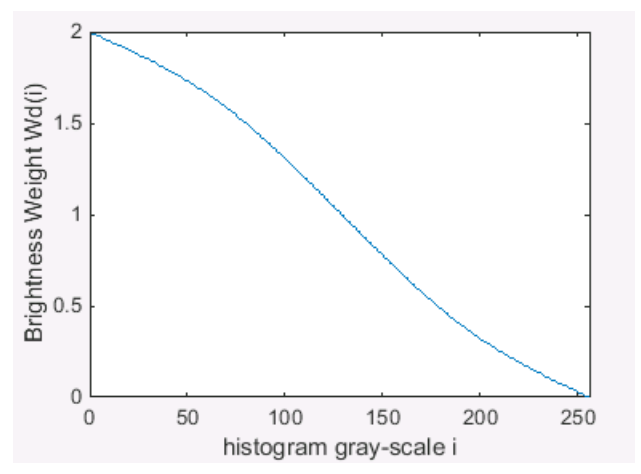

(a) the ambient luminance is darker

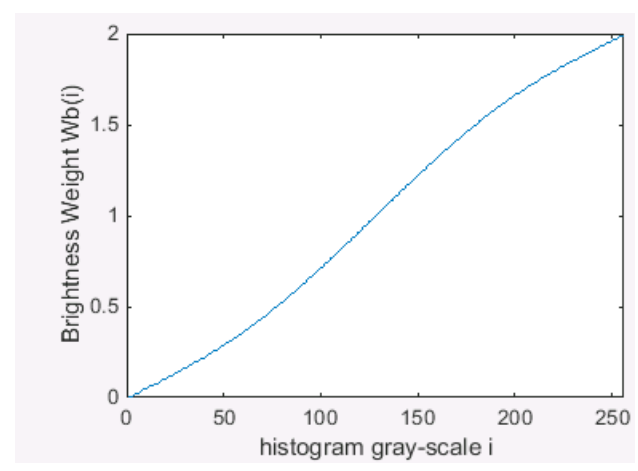

(b) the ambient luminance is brighter

Figure 3. Histogram Mapping Weight Curve in Different Ambient luminance 
According to the ambient luminance $\mathrm{f}_{\text {env }}$, the formula can be used:

$$
\mathrm{W}_{\mathrm{f}}=\mathrm{W}_{\mathrm{d}} * \mathrm{f}_{\text {env }}+\mathrm{W}_{\mathrm{b}} *\left(1-\mathrm{f}_{\text {env }}\right)
$$

Calculate the fitted histogram brightness weight function $\mathrm{W}_{\mathrm{f}}$ as the weight mapping basis for calculating the current image brightness, and then according to:

$$
\mathrm{L}_{\mathrm{f}}=\sum_{i=0}^{255}\left(i * \operatorname{hist}_{(i)} * \mathrm{~W}_{\mathrm{f}(i)}\right) /(\mathrm{h} * \mathrm{w})
$$

Obtain the final brightness value $\mathrm{L}_{\mathrm{f}}$ of the current image. After the above steps, on one hand, we correlate the brightness of the frame image itself with the ambient luminance through the brightness weight $\mathrm{W}_{\mathrm{f}}$ generated by the environmental luminance factor $f_{\text {env }}$ to obtain the mapped image brightness $\mathrm{L}_{f}$; on the other hand, we integrate the image information entropy into the $\mathrm{AE}$ target brightness and calculate the final target brightness $\mathrm{L}_{\text {tar }}$ generated by the combination of the two by using the parameters obtained by big data analysis.

Next, compare $\mathrm{L}_{\mathrm{tar}}$ and $\mathrm{L}_{\mathrm{f}}$, and calculate the exposure deviation $\triangle E X P$ according to the following formula as the adjustment target for the next frame:

$$
\Delta \operatorname{Exp}=\sqrt{\mathrm{L}_{\mathrm{f}} / \mathrm{L}_{\mathrm{tar}}}
$$

Finally, according to the exposure adjustment formula $\Delta \operatorname{Exp}=\Delta_{\mathrm{S}}+\Delta_{\mathrm{GD}}+\Delta_{\mathrm{GA}}$, the increments of exposure time $\mathrm{S}$, digital gain $G_{D}$ and analog gain $G_{A}$ of the camera system are dynamically allocated. The exposure increase is based on the principle of priority $S>G_{A}>G_{D}$; and the exposure decrease is based on the principle of priority $G_{D}>G_{A}>S$.

\section{Comparison Results}

This algorithm has been applied and verified in the security monitoring and intelligent traffic scheme based on Hisilicon 3559A processor and Sony IMX305 image sensor, as shown in Figure 4

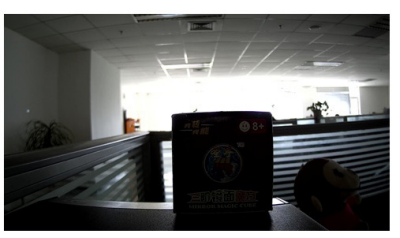

(a)

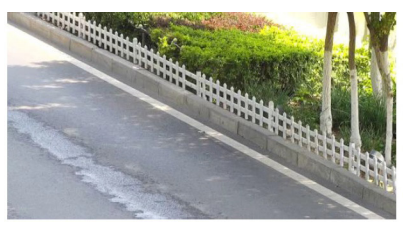

(c)

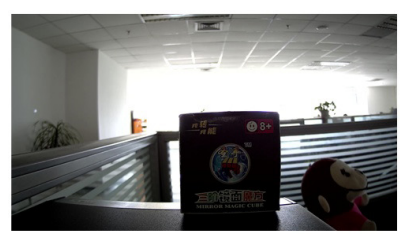

(b)

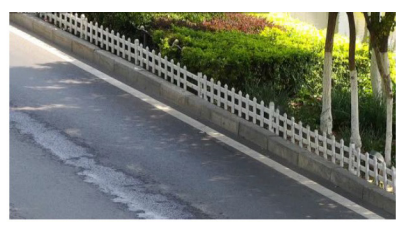

(d)

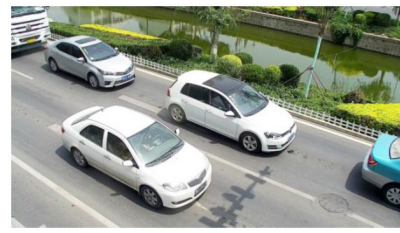

(e)

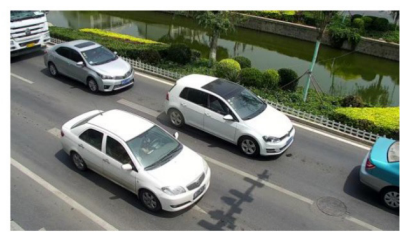

(f)
Figure 4. Comparison of effect between traditional AE algorithm and the algorithm in this paper

(a), (c) and (e) of Figure 4 are effect diagrams of average brightness $\mathrm{AE}$ algorithm, ROI weighted $\mathrm{AE}$ algorithm and information entropy maximization $\mathrm{AE}$ algorithm respectively, (b), (d) and (f) are the effect diagrams of AE algorithm in this paper. By comparing the pictures, it can be seen that the $\mathrm{AE}$ algorithm based on ambient luminance and image information entropy effectively solves the adaptability problems in the traditional AE algorithm, and can obtain better results whether dealing with wide dynamic, high contrast or scenes with rich details.

\section{Conclusion}

This paper proposes a new AE algorithm based on image big data and information entropy. It is the first attempt to combine big data and automatic exposure algorithm, which provides a new direction for the development of $\mathrm{AE}$ algorithm. On one hand, the $\mathrm{AE}$ adjustment direction is guided by the combination of image information entropy and target brightness based on the mining and sorting of image big data; on the other hand, ambient luminance factor is introduced to assist image information statistics; at the same time, the exposure combination factor is dynamically adjusted to achieve the purpose of adjusting the exposure parameters in real time. The algorithm is easy to implement and has strong adaptability to scenes. In actual tests, it solves the adaptability defects of traditional AE algorithm, and can always maintain good image effect regardless of the changes of environment and dynamic range. The algorithm has been widely applied to the frontend acquisition equipment in the field of intelligent security and has broad commercial prospects.

\section{References}

[1] Murakami M, Honda N. An Exposure Control System of Video Cameras Based on Fuzzy Logic Using Color Information[J]. Journal of Japan Society for Fuzzy Theory and Systems, 1996,8 (6):1154-1159. DOI:10.3156/jfuzzy.8.6_172.

[2] Jiayi Liang, Zhiliang Hong. Auto-Exposure Control Algorithm for Large Dynamic Range Scenes[J]. Op- 
toelectronic Engineering, 2008, 35 (5).

DOI:10.3969/j.issn.1003-501X.2008.05.018

[3] Rahman M T, Kehtarnavaz N, Razlighi Q R. Using image entropy maximum for auto exposure[J]. Journal of Electronic Imaging, 2011, 20(1):013007. DOI:10.1117/1.3534855

[4] Yan Chen. Application of Big Data Technology in Image Processing[J]. Electronic Technology and Software Engineering, 2018.

http://kns.cnki.net/kcms/detail/10.1108. TP.20180307.1144.254.html

[5] Qun Ren. Image Processing Technology Based on Information Entropy[J]. Journal of Daqing Normal University, 2017(3).

DOI: 10.13356/j.cnki.jdnu.2095-0063.2017.03.007.
[6] Peiran Li. Research on 3D Reconstruction Based on Video[D].

DOI:CNKI: CDMD: 2.1016.779552

[7] Tianqiang Huang, Tiehao Wu, Hua Zhuo, et al. Method and Device for Detecting Video Frame Insertion Tampering Based on Light Intensity Information $[\mathrm{J}]$. DOI:10.3969/j.issn.1000-3428.2014.07.049

[8] Zepeng Wu, Lingling Guo, Mingchao Zhu, et al. Image Registration Method Combining Image Information Entropy and Feature Points[J]. Infrared and Laser Engineering, 2013 (10).

DOI:10.3969/j.issn.1007-2276.2013.10.046 